\title{
CONSTITUTIVE MODELING AND FINITE ELEMENT SIMULATION OF METAL-POLYMER-METAL SANDWICH STRUCTURES
}

\author{
Pranav Kumar Dileep ${ }^{1 *}$, Stefan Hartmann ${ }^{1}$, Tobias Fischer ${ }^{2}$, Gerhard Ziegmann², Wei \\ Hua $^{3}$, and Heinz Palkowski ${ }^{3}$ \\ ${ }^{1}$ Institute of Applied Mechanics, Clausthal University of Technology, Germany \\ ${ }^{2}$ Institute of Polymer Materials and Plastics Engineering, Clausthal University of Technology, \\ Germany \\ ${ }^{3}$ Institute of Metallurgy, Clausthal University of Technology, Germany \\ *pranav.kumar.dileep@tu-clausthal.de
}

The practical application of light-weight structures in the form of sandwich structures is of particular interest in structural, automotive, marine, and aviation industries. We investigate metal/polymer/metal (MPM) sandwich structures, which show complex response when subjected to metal forming processes due to varying material characteristics within the layers. To evaluate the forming processes of sandwich structures, constitutive models for the face sheets made of heat treated steel of deep drawing quality and the core layer made of PA6 polymer are a prerequisite. For a first instance, a finite strain viscoplasticity model as proposed in $[1,2]$ is chosen. For the core layer, an extended constitutive model as shown in [3] will be proposed. This is based on a thermo-mechanically consistent finite strain overstress-type viscoplasticity model representing the material behavior of PA6. In this regard, a number of experiments are shown, which are the basis of the model. Both constitutive models contain material parameters, which are identified by tensile tests investigating rate-dependent, long term relaxation, and multi-step relaxation behavior performed at room temperature, see for a possible procedure [4]. In the final step, the constitutive models for steel and PA6 are numerically validated using deep drawing and bending experiments of MPM sandwich structures.

\section{References}

[1] Lion, A. (2000). Constitutive modelling in finite thermoviscoplasticity: a physical approach based on nonlinear rheological models. International Journal of Plasticity, 16(5), 469-494.

[2] Hartmann, S., Quint, K. J., and Arnold, M. (2008). On plastic incompressibility within time-adaptive finite elements combined with projection techniques. Computer Methods in Applied Mechanics and Engineering, 198(2), 178-193.

[3] Hartmann, S. (2006). A thermomechanically consistent constitutive model for polyoxymethylene. Archive of Applied Mechanics, 76(5), 349-366.

[4] Krämer, S., Rothe, S., and Hartmann, S. (2015). Homogeneous stress-strain states computed by 3D-stress algorithms of FE-codes: Application to material parameter identification. Engineering with Computers, 31(1), 141-159. 